\title{
On the phase coupling of two components mixing in empirical mode decomposition
}

Article

Accepted Version

Laszuk, D., Cadenas, J. O. and Nasuto, S. J. (2016) On the phase coupling of two components mixing in empirical mode decomposition. Advances in Data Science and Adaptive Analysis, 08 (01). 1650004. ISSN 2424-922X doi: https://doi.org/10.1142/S2424922X16500042 Available at https://centaur.reading.ac.uk/69462/

It is advisable to refer to the publisher's version if you intend to cite from the work. See Guidance on citing.

Published version at: http://dx.doi.org/10.1142/S2424922X16500042

To link to this article DOI: http://dx.doi.org/10.1142/S2424922X16500042

Publisher: World Scientific Publishing

All outputs in CentAUR are protected by Intellectual Property Rights law, including copyright law. Copyright and IPR is retained by the creators or other copyright holders. Terms and conditions for use of this material are defined in the End User Agreement.

\section{www.reading.ac.uk/centaur}

\section{CentAUR}

Central Archive at the University of Reading 
Reading's research outputs online 


\title{
ON THE PHASE COUPLING OF MODE-MIXING TWO COMPONENTS MIXING IN EMPIRICAL MODE DECOMPOSITION
}

\author{
DAWID LASZUK \\ School of Systems Engineering, University of Reading, \\ Reading, RG6 6AY, United Kingdom \\ d.laszuk@pgr.reading.ac.uk \\ http://www.laszukdawid.com \\ JOSE O. CADENAS \\ School of Systems Engineering, University of Reading, \\ Reading, RG6 6AY, United Kingdom \\ o.cadenas@reading.ac.uk \\ SLAWOMIR J. NASUTO \\ School of Systems Engineering, University of Reading, \\ Reading, RG6 6AY, United Kingdom \\ s.j.nasuto@reading.ac.uk \\ Received Day Month Year \\ Revised Day Month Year
}

\begin{abstract}
This paper examines whether or not the mode-mixing investigates frequency mixing effect of Empirical Mode Decomposition (EMD) and whether it can be explained by simple phase coupling between components of the source signal. The source is assumed to be a linear combination of harmonic oscillators. The hypothesis was tested assuming that phases of source signals' components would couple according to Kuramoto's model. Using a Kuramoto's model with as many oscillators as the number of intrinsic mode functions (result of EMD), the model's parameters were adjusted by a particle swarm optimisation (PSO) method. The results show that our hypothesis is plausible, however, a different coupling mechanism than the simple sine-coupling Kuramoto's model are likely to give better results.
\end{abstract}

Keywords: Empirical mode decomposition; instantaneous frequency; frequency mixing ; particle swarm optimisation; Kuramoto model; phase coupling

\section{Introduction}

In recent years, many data-driven decomposition methods have been proposed. One such method is empirical mode decomposition (EMD) [4], which extracts oscillatory components in a general forms a general form. These components are supposed to have significant modes, thus being called intrinsic mode functions (IMFs). In the 
original article, have argued that functions which fulfil IMF properties (discussed in section 2) have well-behaved instantaneous frequencies obtained via the Hilbert transform. The authors call these components physically meaningful, as typically their instantaneous frequencies do not reach negative values and they have modulations in amplitude and frequency.

Due to the absence of a mathematical framework for EMD, it can only be analysed empirically. As it has been observed by many $[5 ; 2 ; 14]$ the decomposition process suffers from the mode-mixing phenomena - , which describes process, when a mode contains more then scale. Another imperfection of EMD is its separation problem. This means, that when acting on a single IMF $(I(t))$ EMD returns the same function, i.e.

$$
I(t) \stackrel{E M D}{\longrightarrow} I(t),
$$

however, when decomposing a signal composed of two or more IMFs $(S(t)=$ $\left.\sum_{i}^{n} I_{i}(t)\right)$ the obtained components do not have to be the same IMFs, i.e.

$$
S(t) \stackrel{E M D}{\longrightarrow}\left\{C_{1}(t), C_{2}(t), \ldots C_{m}(t)\right\},
$$

where there is not necessarily any correspondence between any $I_{i}(t)$ and $C_{j}(t)$. A thorough study of the mode-mixing frequency mixing for two components was presented in [11]. The authors compared IMFs obtained from signals each composed of two cosines, i.e. $S(t)=\cos (t)+a \cos (f t+\phi)$, generated with different amplitudes $a \in \mathbb{R}$ and frequencies $f \in(0,1)$ values. They found that for some $a<0.5$ the goodness of the decomposition, i.e. similarities of IMFs and source components, depends only on the frequency. Moreover, they found, the smaller the value $(f \approx$ $0)$, the better reconstruction of initial modes. They also noted that the transition from almost perfect reconstructions to near impossible is an increasing monotonic function of $f$. This, however, poses a question: what is responsible for mixing when frequencies of input components have similar values? Since only the frequency varies this means that the mixing process depends only on that parameter. As the system is closed, i.e. there are only two components, any added modulation to the first IMF is the same as removing that modulation from the second IMF. Given the results obtained by [11] we hypothesise that the mode mixing phenomena is due to the mutual dependency of instantaneous frequencies of the sources. In order to test this hypothesis, we use harmonic components and see whether coupling between them can reconstruct obtained IMFs. As an interaction model, Kuramoto type phase coupling [8] has been chosen. It assumes that coupling between the oscillators is modulated by the difference of their instantaneous phases.

In this paper, we seek to answer whether mode mixing frequency mixing observed in EMD can be explained by the Kuramoto assumption of coupling between oscillators. The paper is structured as follows: section 2 describes a method for extracting EMD phase, section 3 presents a short introduction to Kuramoto's model and section 4 introduces an optimisation method used to fit parameters in $\mathrm{Ku}$ - 
ramoto's model and the results of the experiments are discussed in 5 . The conclusion is in section 6 .

\section{Huang-Hilbert transform}

There are many methods for phase extraction. The one presented in this paper is based on Huang-Hilbert transform (HHT) [5]. It consists of two steps: first, a signal is decomposed into components with EMD and secondly, the Hilbert transform is performed on each component.

Empirical mode decomposition (EMD) is a data-driven method which extracts oscillatory components [4]. It assumes that a signal is a superposition of amplitudeand frequency-modulated functions, which are referred to as intrinsic mode functions (IMFs). Extraction proceeds iteratively; in each iteration, a slow wave (average of interpolated top and bottom envelopes) is removed, leaving components with slower varying trend. Once the number of zero-crossings and the number of extrema differs by at most one and the trend is close to zero, the function is considered as an IMF. Each IMF is thought to be generated by a different source, which is why only one-to-one correspondences between the IMFs and the reconstructed oscillators is later considered.

EMD lacks a mathematical framework, which makes its results difficult to analyse. This has led many authors to suggest modifications to the original EMD algorithm, such as by using different stopping criterion [5] or by decomposing a modified signal instead, as in Ensemble EMD (EEMD) [15]. Nevertheless, EMD has been successfully applied in many scientific and engineering fields, e.g. [3; 10;6], producing insightful results.

\section{System based on Kuramoto model}

In nature, it is impossible to create an isolated system. Although in most cases the interference has negligible effect, in some closed systems these interferences cannot be avoided. A model which describes interactions between oscillators has been proposed by 8 . He observed that the change in oscillators' periods can be explained by assuming coupling in the phase domain. Each oscillator has an intrinsic frequency and its observed frequency modulated by the difference between each pair of phases [13]. Mathematical form of the model for $i^{\text {th }}$ oscillator is given as

$$
\dot{\theta}_{i}=\omega_{i}+\sum_{j=1}^{N} k_{i, j} \sin \left(\theta_{j}-\theta_{i}\right)
$$

where $\dot{\square}$ denotes the time derivative, $\omega_{i}$ is the intrinsic frequency and $k_{i, j}$ are coupling strength parameters. If there is no coupling, i.e. when all $k_{i, j}=0$, then the oscillators behave as simple, harmonic oscillators.

Solving these coupled differential equations requires $N$ initial phase values, $N$ intrinsic frequencies and $N(N-1)$ values for coupling strengths parameters. In 
total, $N(N+1)$ parameters describe a system and these parameters have to be estimated from data.

\section{Parameter estimation}

Our main assumption is that the measured signal is a linear combination of a few interacting oscillators. These components are unique in the frequency domain, that is, their intrinsic frequencies are not similar. With EMD one should be able to extract these components and with the use of the Hilbert transform one can estimate phases.

Particle swarm optimisation (PSO) [7] is an optimisation method where many agents search through a parameter space in an iterative manner. They interact with each other by announcing their fitness and their position. Movement of $i^{\text {th }}$ particle is dictated by the formula

$$
\begin{aligned}
\vec{V}_{i}(t)=\phi_{V} \vec{V}_{i}(t-1) & +\phi_{L}\left(\vec{B}_{i}(t)-\vec{X}_{i}(t)\right) \\
& +\phi_{G}\left(\vec{G}(t)-\vec{X}_{i}(t)\right),
\end{aligned}
$$

where $X_{i}, V_{i}$ and $B_{i}$ are the particle's current position, its velocity and its best position until time $t$ respectively. $G$ is the best global position discovered by any particle until time $t$. Communication is performed after each iteration, when all particles update their positions based on the velocity, i.e. $X(t+1)=X(t)+V(t)$. At $t=0$ all particles have a randomly chosen position and velocity.

In the experiments described below, swarms consisted of 400 particles. Their sizes were chosen arbitrary big to insure greater search region. Each particle describes a system mentioned in previous section. Each particle is a 6 dimensional vector consisting of all initial values, i.e. $X_{i}(0)=\left[\theta_{01}, \theta_{02}, \omega_{1}, \omega_{2}, k_{1,2}, k_{2,1}\right]$, which fully describe a system mentioned in previous section. The intrinsic frequencies for the oscillators were drawn from Gaussian distributions, where the expected values and standard deviations were equal to those of the IMFs' instantaneous frequencies. Phases and values of $k$ couplings were also drawn from Gaussian distributions, however their absolute values were used. The expected values and standard deviations were $\pi$ and $\pi / 4$ for phases, and 0 and 5 for $k$ values.

The optimising fitness function is given as

$$
M=\sqrt{\frac{1}{N} \frac{1}{T} \sum_{n=1}^{N}\left(\sum_{t=1}^{T}\left(\dot{\Theta}_{n}(t)-\dot{\theta}_{n}(t)\right)^{2}\right)},
$$

where $\Theta_{n}$ and $\theta_{n}$ are phases of $n^{\text {th }}$ IMF and reconstructed oscillator respectively. Summation goes through all $t$ timestamps and there are $N$ oscillators of length $T$ time points. The optimisation procedure terminates when, after 100 initial iterations, the cost value is the same for 20 consecutive iterations. 


\section{Experiments}

In order to show phase coupling between components of source signal, a set of experiments was conducted. They all were based on synthetic data constructed according to the formula

$$
S_{f}(t)=\cos (13 \cdot 2 \pi t)+2 \cos (f \cdot 2 \pi t+\phi),
$$

i.e. where frequency $(f \in[1,7]$ with step 0.5$)$ and initial phase $(\phi \in[0,2 \pi]$ with step

$\frac{2 \pi}{15}$ ) of the second component were varied. For each $f$ and $\phi$ combination EMD was performed many times with multiple spline techniques (natural cubic or Akima [1] spline) and parameters related to stopping criteria [12]. Out of these options a set was chosen that minimises the metric $M=\sqrt{M_{1}^{2}+M_{2}^{2}}[?]$, where $M_{1}$ quantifies the pairwise crossing overs of instantaneous frequencies between IMFs, and $M_{2}$, which penalises based on the overlap between IMF's amplitude and phase spectra ${ }^{\mathrm{a}}$. After the decomposition was performed, the Hilbert transform of each IMF was used in order to obtain its instantaneous phase and amplitude. Due to the error created by boundary effects, each component's first and last $0.5 \mathrm{~s}$ had to be removed leaving $2 \mathrm{~s}$ of signal.

Exemplary decomposition obtained for $f=4$ is presented in Figure 1 . The top graph contains input signal, whereas the second and the third rows are respectively first and second IMFs. Each component (solid line) was scaled (scale in top left corner) so that its maximum value was one. On the same figure, dashed lines were used to display cosine function of instantaneous phased $(\cos (\Phi(t)))$ obtained via the Hilbert's transformation for corresponding IMF. Almost complete overlap suggests, that there is very little modulation in the amplitude. For this reason in further analysis only phase modulations are considered.

In order to better understand what modulations are present in instantaneous frequencies of the IMF, the Fourier transform was computed for each $f$. The results were scaled such that the largest value is one. If there were no modulations in instantaneous frequency, one would expect zero-valued spectrum everywhere except for frequency 0. Contrary, a predominant periodic modulation would manifest itself as a single spike in the Fourier spectrum. Figure 2 displays the spectra for the first IMF for all $f$. In the figure, the values of the Fourier frequencies were shifted by value $\Delta_{f}=13 \quad f$. This step was performed in order to align the peaks, which ean be seen on the top and back projections. In the figure, one can observe that peaks are aligned. This is additionally emphasised by overlapping results with a line $(F=13-f)$. Similar results are visible for the second IMF, presented in Figure 3. However, in this case peaks are aligned along a different line, thus frequencies were shifted with function $\Delta_{f}=2(13 \quad f)$. However, in this case peaks are aligned along a different line, i.e. $F=2 \cdot(13-f)$

The highlighted peaks presented in both figures (Fig. 2 and Fig. 3) can also be ebserved on cress correlation be all pairs of IMFs. The highlighted dependency

\footnotetext{
aSource code used in this analysis is available to download from one of the author's webpage [9]
} 

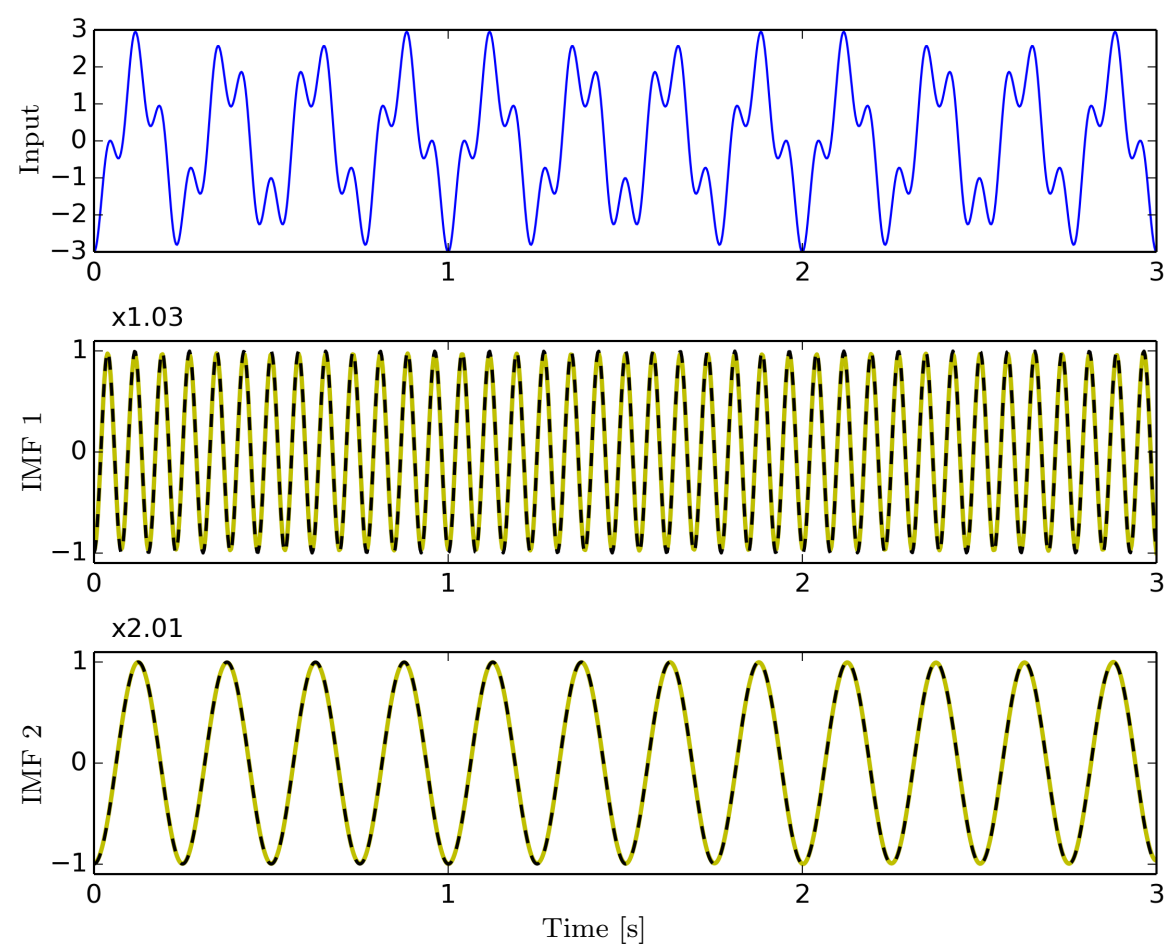

Fig. 1. Synthetic signal (top row) generated for $f=4$ and its EMD decomposition. IMFs (solid line) are scaled (scale in top left corner) so that their maximum value is 1 . For comparison dashed lines present cosine functions of IMF's instantaneous phases.

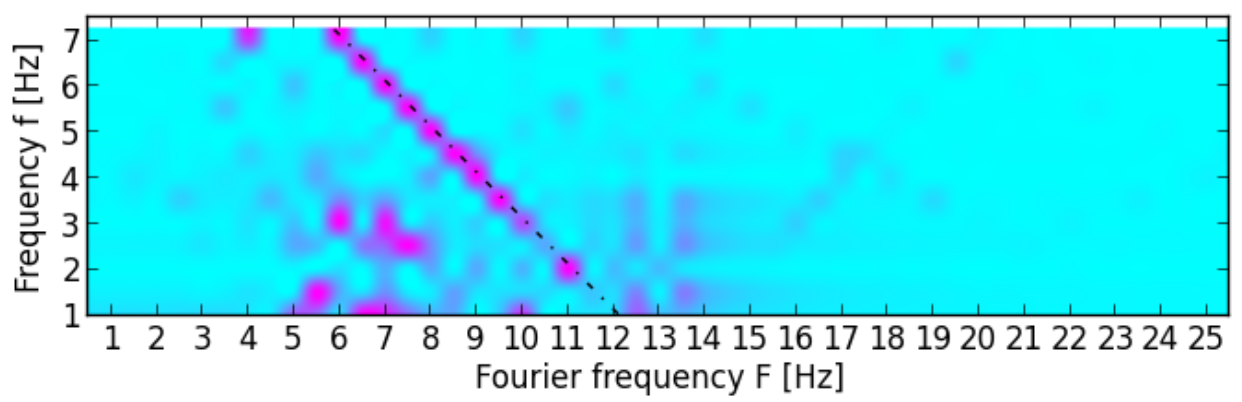

Fig. 2. Fourier spectra obtained for the first IMF for different values of frequency $f$ (Eq. (6)). For each $f$, all values were scaled to the maximum being one. Dashed line highlights trend dictated by function $F=13-f$.

between peaks of Fourier spectra and initial frequency $f$ can also be observed when analysing cross-correlations between all pairs of IMFs' instantaneous frequencies. 


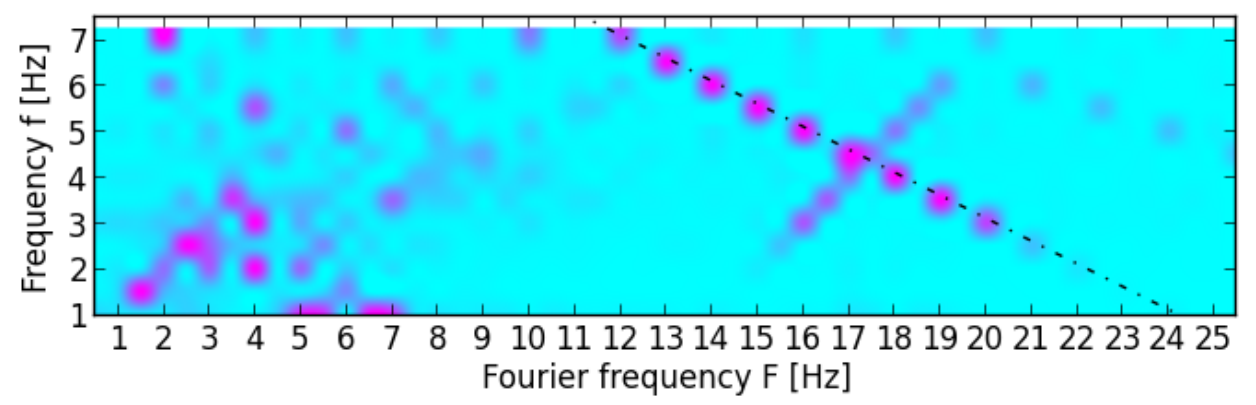

Fig. 3. Fourier spectra obtained for the second IMF for different values of frequency $f$ (Eq. (6)). For each $f$, all values were scaled to the maximum being one. Dashed line highlights trend dictated by function $F=2 \cdot(13-f)$.

Such analysis emphasise modulations common for both components. Since there are only two IMFs, those modulations must be a product of their interaction, most likely a mixing of frequencies. A singular example is shown in Fig. 4, where the top plots represent the instantaneous frequencies centred at zero by mean subtraction. On the same figure, the middle graph presents cross-correlation between the instantaneous frequencies of both IMFs, whereas the bottom graph shows the Fourier spectrum of the correlation signal. The vertical line indicates the value equal to the difference of the IMFs' mean frequencies, which for this example it is $\Delta f=13 \mathrm{~Hz}-4 \mathrm{~Hz}=$ $9 \mathrm{~Hz}$. Cumulative result for all pairs of IMFs is shown in Figure 5, where for each $f$ the Fourier spectrum of the IMFs' correlations is plotted. Each spectrum was normalised, so that the biggest value is one. This step allows for visual comparison of the results, as the maximum amplitude of cross-correlation depends on the $f$ value and varies by a factor of $10^{5}$ when comparing results for $f=1 \mathrm{~Hz}$ and $f=7$ Hz. Again it can be observed that there exist two channels of peaks along lines $F_{1}=13-f$ and its harmonic $F_{2}=2 \cdot(13-f)$. This suggests strong coupling between the instantaneous frequencies of the IMFs at some $f$.

A quantitative attempt to explain visible effects was performed by fitting $\mathrm{Ku}$ ramoto coupling model to the obtained instantaneous frequencies. An example of the reconstruction is presented in Figure 6. On this graph, the left column contains the instantaneous frequency of the IMF (solid line) and the reconstructed one via Kuramoto model (dashed line). The right column shows the difference between the two instantaneous frequencies for each IMF, which are first and second for top and bottom rows respectively. The values of obtained parameters and measure of fitness (Eq. (5)) are presented in Table 1. Parameters $f_{1}$ and $f_{2}$ relate to the intrinsic frequencies of Kuramoto model for the first and the second IMF respectively and it can be seen that they match relatively close to the input signal's modes. For small values of $f$, i.e. when there is a big difference in frequencies between input components, there is little coupling, i.e. $k_{1}$ and $k_{2}$ are small and they increase with $f$. 

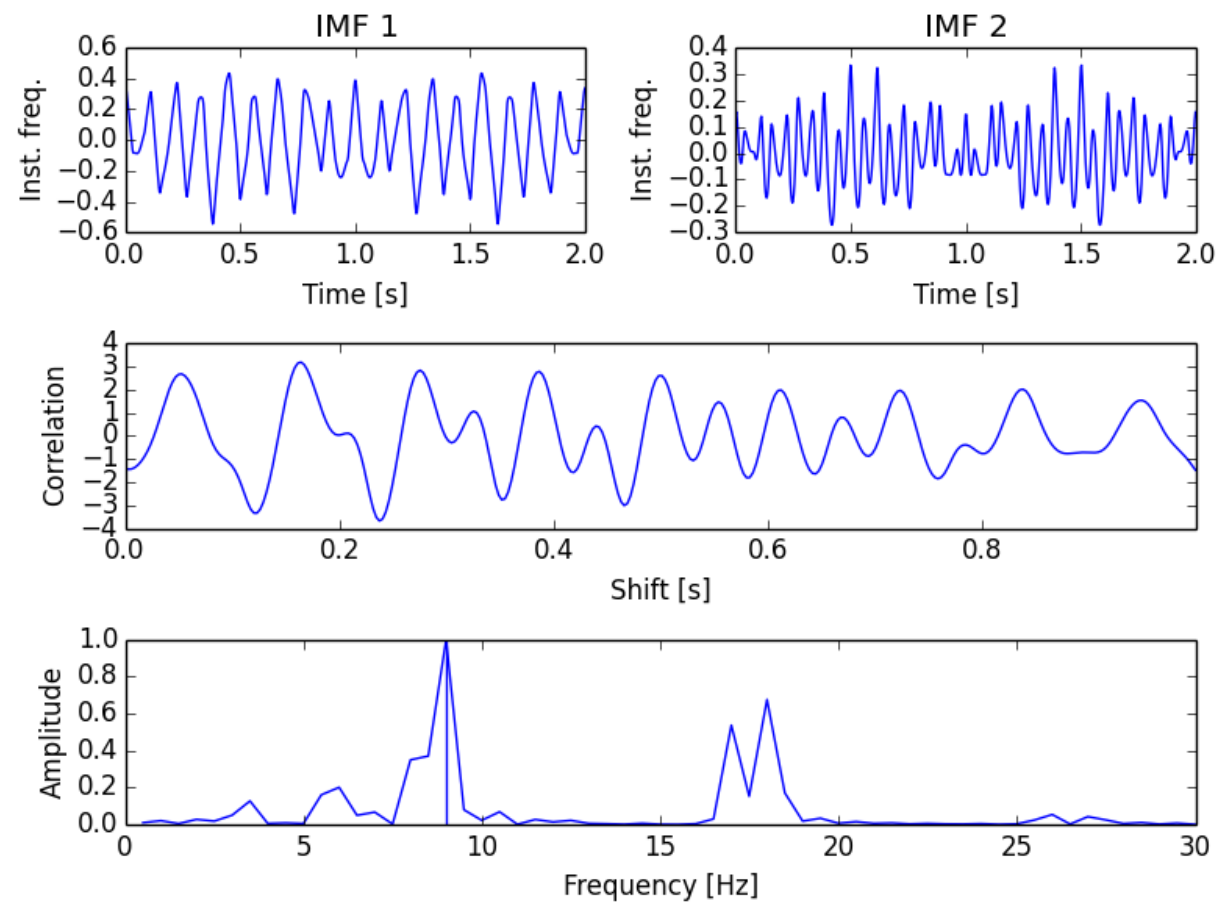

Fig. 4. Correlation between IMFs' instantaneous frequencies centered at zero (case when $f=4$ ). Top plots represent the instantaneous frequencies, middle plot displays their cross-correlation and the bottom graph shows its Fourier spectrum. Vertical line marks value equal to the difference of IMFs' mean frequencies.

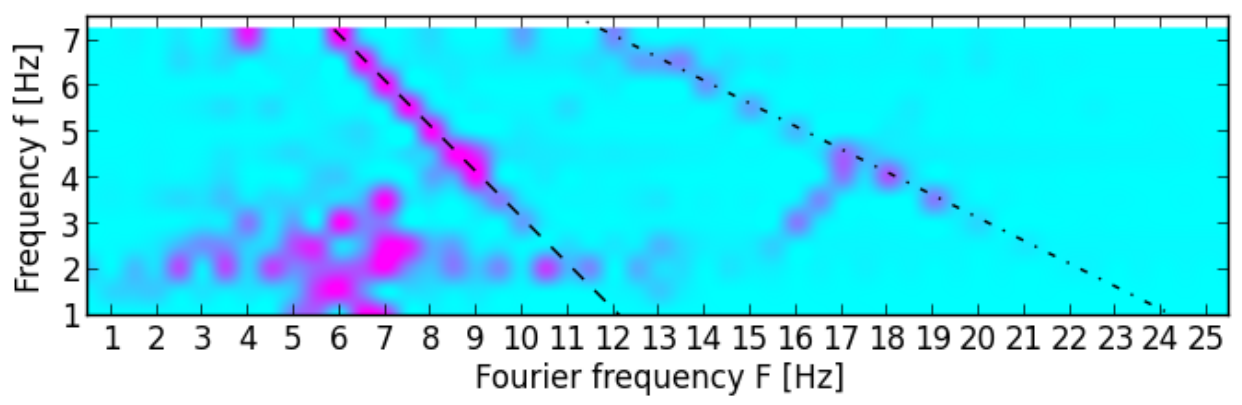

Fig. 5. Fourier transform $F$ of correlation function between each pair of IMFs for different values of frequency $f$. All values were normalised, such that for given $f$ the maximum is equal to one. Additionally, on the top projection of the figure two lines have been drawn $-F_{1}=13-f$ (dashed line) and $F_{2}=2 \cdot(13-f)$ (dash-dotted line). 
Table 1. Parameters obtained for Kuramoto's model to fit the data for different input modes. Frequencies $f_{1}$ and $f_{2}$ refer to the mean instantaneous frequencies, $k_{1}$ and $k_{2}$ are the coupling values, and $M_{r}$ and $M_{w}$ refer to fitness (see Eq. 5) with and without couplings. Indices 1 and 2 refer to the first and the second IMFs respectively.

\begin{tabular}{l|cccc|ccc}
$\mathrm{f}$ & $f_{1}$ & $f_{2}$ & $\left|k_{1}\right|$ & $\left|k_{2}\right|$ & $M_{r}$ & $M_{w}$ & $\left(M_{w}-M_{r}\right) / M_{w}[\%]$ \\
\hline 1 & 13.000 & 1.000 & 0.001 & 0.000 & 0.020 & 0.020 & 0.01 \\
1.5 & 13.013 & 1.501 & 0.031 & 0.000 & 0.069 & 0.069 & 0.07 \\
2 & 13.013 & 2.002 & 0.696 & 0.004 & 0.250 & 0.337 & 25.88 \\
2.5 & 13.013 & 2.502 & 0.003 & 0.000 & 0.081 & 0.081 & 0.10 \\
3 & 13.013 & 3.003 & 0.046 & 0.009 & 0.082 & 0.084 & 2.81 \\
3.5 & 13.013 & 3.504 & 0.180 & 0.020 & 0.076 & 0.097 & 21.48 \\
4 & 13.001 & 4.000 & 0.273 & 0.031 & 0.087 & 0.133 & 34.96 \\
4.5 & 13.013 & 4.504 & 0.509 & 0.027 & 0.150 & 0.227 & 33.96 \\
5 & 13.005 & 5.011 & 2.225 & 0.023 & 0.300 & 0.950 & 68.41 \\
5.5 & 13.027 & 5.511 & 2.162 & 0.178 & 0.364 & 0.878 & 58.50 \\
6 & 13.030 & 5.989 & 5.687 & 0.036 & 0.700 & 2.203 & 68.24 \\
6.5 & 13.006 & 6.492 & 2.411 & 0.014 & 0.442 & 0.962 & 54.07 \\
7 & 13.044 & 7.021 & 4.930 & 0.164 & 2.285 & 3.280 & 30.35
\end{tabular}

However, increasing $k$ does not necessarily decrease the fit. $M_{r}$ and $M_{w}$ refer to the mean square error (Eq. (5)) of the fit of Kuramoto model of IMFs' instantaneous frequencies with coupling $\left(M_{r}\right)$ and without coupling $\left(M_{w}\right)$. The last column represents how much percentage-wise the reconstruction explains the variation. It can be seen that for the small values of $f_{2}$, i.e. where $f_{2} \leq 3$, the mean square error has not decreased significantly. The reason is that IMFs almost perfectly match the source components and there is no need to include coupling $k$ factors. Special case is $f=2$ for which coupling $k_{1}$ is relatively large and the error is decreased by $25 \%$. In can be observed that for all examples, the coupling $k_{1}$ is bigger than $k_{2}$. This means that instantaneous frequency of the first IMF has more modulation proportional to the difference of the source's frequencies. This is in accordance with Figures 2 and 3, where it can be seen that predominant modes frequencies in instantaneous frequencies are $\Delta_{f 1}=13-f$ and $\Delta_{f 2}=2 \cdot(13-f)$ for the first and the second IMFs respectively.

\section{Conclusion}

As observed in all the experiments in section 5, the instantaneous frequency of each IMF has some modulations. These modulations, both their amplitudes and frequencies, seem to depend on the difference between the input's modes. This means that there is some interaction between the components, which depends on their frequencies and phases. An attempt to explain this mode-mixing frequency mixing was performed by assuming Kuramoto type phase coupling between the modes that is proportional to sine of their differences. In many cases the fit was 

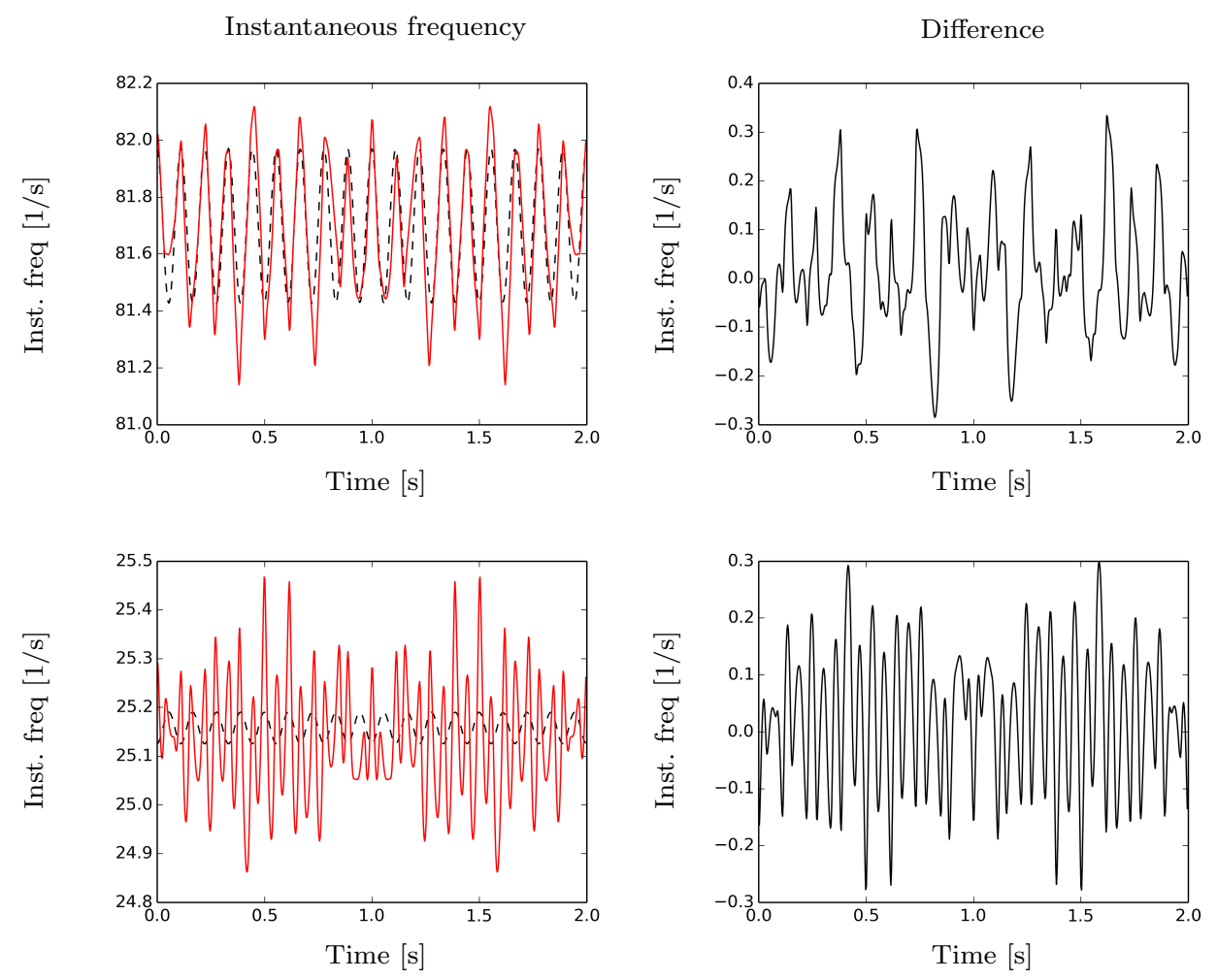

Fig. 6. Comparison of instantaneous frequencies for $f=4$. Left column contains IMF's instantaneous frequency (solid line) and the reconstructed one (dashed line), whereas the right column shows their differences. Top and bottom rows correspond to first and second IMFs respectively.

good (see Table 1). However, in some cases including the coupling only reduced the error of fit by $20 \%$. This implies that there is more complex behaviour between the modes than a simple sine coupling. A possible solution would be to allow for additional components in Kuramoto's coupling function, e.g. including harmonic modulations in Eq. (3). Our future work will focus on modifying the mechanism of coupling functions seeking for a better fit to experimental data. 


\section{References}

Hiroshi Akima. A New Method of Interpolation and Smooth Curve Fitting Based on Local Procedures. Journal of the ACM, 17(4):589-602, October 1970.

Ryan Deering and JF James F. Kaiser. The Use of a Masking Signal to Improve Empirical Mode Decomposition. In Proceedings. (ICASSP '05). IEEE International Conference on Acoustics, Speech, and Signal Processing, 2005., volume 4, pages 485-488. IEEE, 2005.

Alp Erturk, Mehmet Kemal Gullu, and Sarp Erturk. Hyperspectral Image Classification Using Empirical Mode Decomposition With Spectral Gradient Enhancement. IEEE Transactions on Geoscience and Remote Sensing, 51(5):2787-2798, May 2013.

Norden E. Huang, Zheng Shen, Steven R. Long, Manli C. Wu, Hsing H. Shih, Quanan Zheng, Nai-Chyuan Yen, Chi Chao Tung, and Henry H. Liu. The empirical mode decomposition and the Hilbert spectrum for nonlinear and non-stationary time series analysis. Proceedings of the Royal Society A: Mathematical, Physical and Engineering Sciences, 454(1971):903-995, March 1998.

Norden E. Huang, M.-L. C. Wu, S. R. Long, S. S. P. Shen, W. Qu, P. Gloersen, and K. L. Fan. A confidence limit for the empirical mode decomposition and Hilbert spectral analysis. Proceedings of the Royal Society A: Mathematical, Physical and Engineering Sciences, 459(2037):2317-2345, September 2003.

Norden E. Huang and Zhaohua Wu. A review on Hilbert-Huang transform: Method and its applications to geophysical studies. Reviews of Geophysics, 46(2):RG2006, June 2008.

J Kennedy and R Eberhart. Particle swarm optimization. Neural Networks, 1995. Proceedings., IEEE International Conference on, 4:1942-1948 vol.4, 1995.

Yoshiki Kuramoto. Chemical Oscillations, Waves, and Turbulence, volume 19 of Springer Series in Synergetics. Springer Berlin Heidelberg, Berlin, Heidelberg, 1984.

Dawid Laszuk. Python implemention of Empirical Mode Decomposition algorithm, 2014-.

Shih-Lin Lin, Pi-Cheng Tung, and Norden E. Huang. Data analysis using a combination of independent component analysis and empirical mode decomposition. Physical Review E, 79(6):066705, June 2009.

Gabriel Rilling and Patrick Flandrin. One or Two Frequencies? The Empirical Mode Decomposition Answers. IEEE Transactions on Signal Processing, 56(1):8595, January 2008.

Gabriel Rilling, Patrick Flandrin, Paulo Goncalves, et al. On empirical mode decomposition and its algorithms. In IEEE-EURASIP workshop on nonlinear signal and image processing, volume 3, pages 8-11. NSIP-03, Grado (I), 2003.

Steven H. Strogatz. From Kuramoto to Crawford: exploring the onset of synchronization in populations of coupled oscillators. Physica D: Nonlinear Phenomena, 143(1-4):1-20, 2000. 
Baoping Tang, Shaojiang Dong, and Tao Song. Method for eliminating mode mixing of empirical mode decomposition based on the revised blind source separation. Signal Processing, 92(1):248-258, January 2012.

Zhaohua Wu and Norden E. Huang. Ensemble Empirical Mode Decomposition: a Noise-assisted Data Analysis Method. Advances in Adaptive Data Analysis, 1(1):1-41, January 2009. 